\title{
A Directional Cognitive-Radio-Aware MAC Protocol for Cognitive Radio Sensor Networks
}

\author{
Han $\mathrm{Su}^{1}$ and Sangman $\mathrm{Moh}^{2}$ \\ ${ }^{1}$ China Post, Suzhou, China \\ ${ }^{2}$ Dept. of Computer Eng., Chosun Univ., Gwangju, Korea \\ hjtswg@126.com,smmoh@chosun.ac.kr
}

\begin{abstract}
Recently, cognitive radio $(C R)$ technology is broadly applied even to sensor networks. Cognitive radio sensor networks (CRSNs) have been studied and, apparently, sensor nodes equipped with directional antennas achieve higher energy efficiency. This paper proposes a medium access control (MAC) protocol named directional CR-aware MAC (DC-MAC) for CRSNs. To the best of the authors' knowledge, this is the first work to propose a MAC protocol for CRSNs with directional antennas. The simulation study shows that DC-MAC is more energy efficient than the conventional protocol by 50\%-60\% for the RMF TR 1001 transceiver and by 20\%-50\% for the Bluetooth transceiver.
\end{abstract}

Keywords: Cognitive radio sensor network, cognitive radio, directional antenna, medium access control, energy efficiency

\section{Introduction}

The usage of spectrum resources and the regulation of radio emissions are coordinated by national regulatory bodies such as the Federal Communications Commission (FCC). The FCC assigns spectrum to license holders. In cognitive radio (CR) networks, the license holders are known as primary users (PUs). On the other hand, the users that opportunistically use the licensed spectrum are called secondary users (SUs). The recent study on the actual spectrum utilization shows that a major portion of the licensed band experiences low utilization [1-4]. Therefore, CR becomes a promising technology in wireless communications [5]. As the PUs have priority in using the licensed spectrum, the SUs should not interfere with the PUs. Thus, the SUs have to pay attention to the PUs' behavior as well as to the operational environment.

Wireless sensor networks (WSNs) are widely used in environment monitoring, smart spaces, medical systems, and many other areas. In such networks, sensor nodes are battery-operated and energy efficiency is one of the most important design criteria because it determines network lifetime [6]. Cognitive radio sensor networks (CRSNs) have been studied recently due to the spectrum scarcity problem. Directional antennas can be effectively employed in CRSNs because they inherently save energy. They can also increase the transmission range and reduce interference by beamforming the radio toward a desired direction with higher spatial reuse and better link reliability, which could significantly increase the network capacity [7].

As in WSNs, a good medium access control (MAC) protocol must be energy-efficient in CRSNs. This is mainly because it is impossible or very difficult to recharge the battery of sensor nodes.

Many energy-efficient MAC protocols have been proposed for general WSNs. The most famous one is sensor MAC (S-MAC) [6]. Furthermore, [8, 9] are extensions of SMAC. To effectively save energy, a clustering mechanism is employed in WSNs. The clustering mechanism in low-energy adaptive clustering hierarchy (LEACH) [10] is widely accepted in many WSNs. In clustered WSNs, many-to-one (i.e., sensor nodes to 
their cluster heads and cluster heads to the sink) communication is dominant and should be effectively exploited in the design of a MAC protocol.

Many researchers have attempted to design MAC protocols for CR networks [11-17]. They can be classified according to their architectures or behaviors. In [11], the authors propose a multichannel parallel transmission scheme with multichannel assignment and multichannel contention. In [12], the authors exploit a mathematic approach to avoid SU interference with a PU. Distance-dependent MAC (DDMAC) [13] attempts to achieve high throughput with a suboptimal channel assignment algorithm. Ad-hoc secondary-user MAC (AS-MAC) [14] is based on time-division multiple access/ frequency-division multiple access (TDMA/FDMA) in a global system for mobile communications (GSM) network. It uses time slots to obtain higher spectrum utilization. In [15], a cross-layer multichannel MAC is proposed, which enables the SUs to identify and use the available frequency spectrum without causing harmful interference to the PUs. Multichannel cognitive radio MAC (MCR-MAC) [16] is similar to AS-MAC, and it divides the spectrum into many time slots. Dynamic de-centralized hybrid MAC (DDH-MAC) [17] is a hybrid of both global common control channel (GCCC) and non-GCCC MAC protocols in order to increase the performance and security.

Directional MAC (DMAC) [7] is the pioneering MAC protocol in directional antenna systems. DMAC increases the spatial reuse and throughput in comparison to carrier sense multiple access with collision avoidance (CSMA/CA). However, it has some other problems such as deafness. In [18], a MAC protocol that addresses the deafness problem while using directional antennas is proposed.

This work is motivated by the fact that no MAC protocol has been proposed for CRSNs with directional antennas. In this paper, we present an energy-efficient MAC protocol for CRSNs with directional antennas named directional CR-aware MAC (DCMAC). To the best of the authors' knowledge, no study on MAC for CRSNs with directional antennas has been reported in the literature. So, the proposed DC-MAC is compared to the conventional MAC protocol for CRSNs with omnidirectional antennas. According to our simulation study, DC-MAC is more energy efficient than the conventional protocol by 50\%-60\% for the RMF TR 1001 transceiver and by $20 \%-50 \%$ for the Bluetooth transceiver.

The rest of this paper is organized as follows: In the following section, the important issues in association with directional antennas are introduced with comprehensive examples. In Section 3, the proposed DC-MAC protocol is presented in detail in terms of concept and operation. In Section 4, the energy efficiency of the proposed DC-MAC is evaluated via simulation and then compared with the conventional protocol. Finally, this paper is concluded in Section 5.

\section{Issues with Directional Antennas}

There is no doubt that the network throughput can be improved by the use of directional antennas. The use of directional antennas also provides wider coverage range and reduced power consumption. However, there are some access problems that come into existence with the use of directional antennas. In this section, we describe the important MAC issues associated with the use of directional antennas.

\subsection{New Hidden Terminal Problem}

The directional transmission of RTS/CTS may lead to two additional hidden terminal problems due to the directional antennas: (i) hidden terminal due to asymmetry in gain and (ii) hidden terminal due to unheard RTS/CTS.

If any one or both of the two nodes are in omni-directional mode and out of range, then they both may fall within the range which might cause collision when they beam-form towards each other [21]. In Figure 1, two nodes $\mathrm{X}$ and $\mathrm{Y}$ are communicating directionally 
with gain $G^{d}$ towards each other. $Z$ listening omni-directionally with gain $G^{o}$ is unaware of this communication. If $Z$ has a packet to deliver to $X$, it changes to directional mode and does carrier sensing towards $X$ with gain $G^{d}$ and finds the channel to be idle. Now, as both $\mathrm{Z}$ and $\mathrm{Y}$ are beam-formed towards $\mathrm{X}$ with gain $\mathrm{G}^{\mathrm{d}}$, there are high chances for the frame sent by $\mathrm{Z}$ to interfere the ongoing communication between $\mathrm{X}$ and $\mathrm{Y}[18]$.

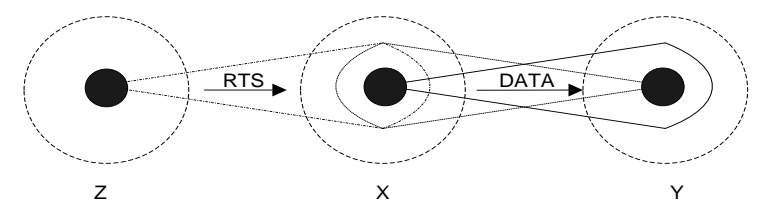

Figure 1. Hidden Terminal Due to Asymmetry in Gain

This problem arises when $\mathrm{P}$ is communicating with $\mathrm{Q}$ directionally as shown in Figure 2. $\mathrm{R}$ and $\mathrm{S}$ are two other nodes in the vicinity of $\mathrm{P}$. While $\mathrm{P}$ is beam-formed towards $\mathrm{Q}, \mathrm{R}$ gets a packet destined to S. Now the RTS/CTS handshake between R and S is unheard by $\mathrm{P}$. Later on finishing the communication with $\mathrm{Q}, \mathrm{P}$ (that is unaware of the ongoing communication between $\mathrm{R}$ and $\mathrm{S}$ ) gets a packet destined to $\mathrm{R}$. Thus, node P's transmission may cause collision on the ongoing communication between $\mathrm{R}$ and $\mathrm{S}$ [18].

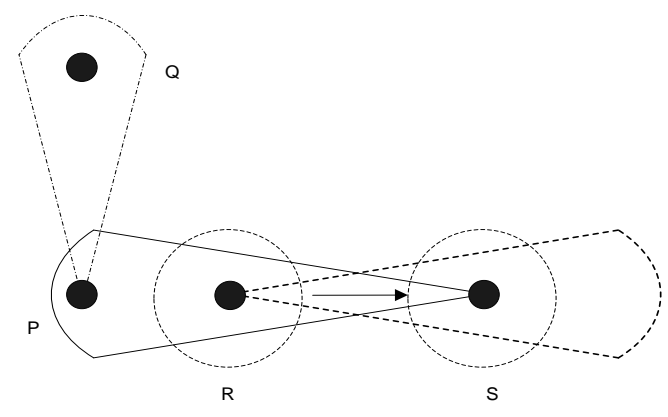

Figure 2. Hidden Terminal due to Unheard RTS/CTS

\subsection{Deafness}

This problem arises when the intended receiver is unable to receive a packet due to the antenna gain pattern $[7,18]$. Let us consider $P, Q, R$ and $S$ nodes in Figure 3. $P$ has a packet destined for $S$. $P$ communicates with $S$ through $R$. Thus, $R$ beam-forms in the direction of $S$. As the communication between $R$ and $S$ is going on, $Q$ or $P$ may have packets for $R$. But, as $R$ is in directional communication with $S, Q$ or $P$ cannot establish the communication. They assume congestion to be the cause of failure and long back-off. Then, after multiple failures and/or back-offs, the back-off duration increases. This causes node $P$ or $Q$ to wait until the back-off expires before the next attempt to retransmit even after $S$ finishes its communication.

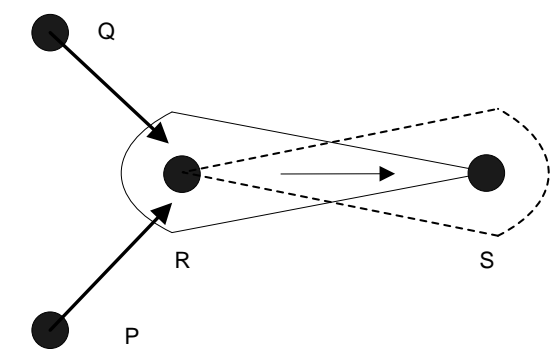

Figure 3. A Scenario of Deafness 


\subsection{Blocking Problem}

The MAC layer makes use of FIFO queues in order to manage the packets to be sent through the shared medium. This scheme introduces head of line (HoL) blocking problem for the protocols using directional antennas. If a packet at the top of the queue is blocked because the medium is not free in that direction, then other packets which are in the queue with some other directions are also blocked [21]. For example, we can see in Figure 4 that node $P$ has packets for $Q, R$ and $S . S$ and $T$ are in communication with each other. So, when a packet for $S$ is at the top of the queue, $P$ has to wait until the medium is free. This causes unnecessary waiting for $Q$ and $R$.

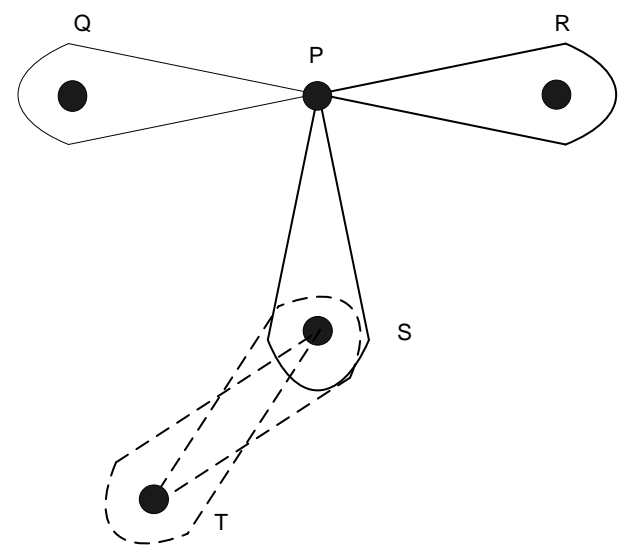

Figure 4. A Scenario of the HoL Blocking Problem

\subsection{Neighbor Location and Discovery}

The information about the location of neighbours is vital in case of directional antennas to enhance the link quality. This information helps the transmitting nodes to beam-form in the direction of the receiver so that the gain is maximized [21]. Exact location information reduces the effect of interference from other nodes to a large extent.

Devices such as GPS are used in nodes to determine the location of the neighbours. GPS is costly and doesn't work in indoor environments. These limitation refrain GPS from easy usability. Various schemes have been proposed to solve the issue of neighbour discovery. Actually, neighbour discovery must be supported with the direction of arrival information. So, for simplicity, many existing protocols assume that the positions of neighbours are known ahead.

\section{Directional CR-Aware MAC Protocol}

In this section, we present our proposed DC-MAC protocol in detail. A switched beam antenna is assumed in our study. The antenna can operate either in directional mode or in omnidirectional mode. The switched beam antenna is equipped with eight sectors. When the antenna operates in the directional mode, it chooses one of the eight sectors and transmits signals in that direction. When the antenna operates in the omnidirectional mode, it transmits or receives signals in all directions. It is also assumed that the power of directional antennas can be adjusted and the transmission range in the directional mode is the same as that in the omnidirectional mode.

In our study, each PU uses its licensed band, and multiple channels are available for each licensed band. There is also one unlicensed band such as the industrial, scientific, and medical (ISM) band, which is used for exchanging the control messages. Every sensor node maintains a channel utilization table for the licensed bands and updates it 
continuously. The channel utilization table consists of a licensed band, channel identifier, and measured channel utilization.

The communication between nodes is performed according to four-way handshaking. In other words, the four frames of directional RTS (Ready To Send), omnidirectional CTS (Clear To Send), directional data, and directional ACK (ACKnowledgement) are exchanged in sequence between the sender and the receiver. Figure 5 shows the four-way handshaking between the sender and the receiver, where DIFS and SIFS represent the distributed coordination function (DCF) interframe space and short interframe space, respectively.

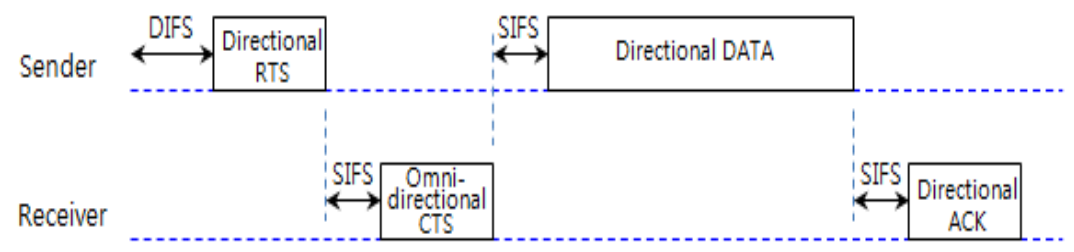

Figure 5. Four-Way Handshaking between a Sender and a Receiver

A sensor node attempts to communicate with its cluster head and it checks the channel utilization table and selects a channel with the lowest utilization. The node then senses the channel. When the channel is not idle, the node senses the second lowest utilized channel. Also, the sender negotiates with the receiver through a control channel. The sender sends RTS with the information of the licensed band ID, channel ID, and expected transmission time. The receiver replies with CTS with the information of the licensed band ID, channel ID, and expected transmission time. Here, the expected transmission time is broadcasted for the neighboring nodes to adjust their network allocation vector (NAV). Both the sender and the receiver switch their band to the selected channel and start a transmission. Unlike the conventional RTS-CTS-DATA-ACK sequence for a transmission, we divide a data packet into multiple small data fragments. Thus, we use RTS-CTS-Fragment1Fragment2-Fragment3-ACK1-Fragment4-...-ACKn for a transmission as shown in Figure 6. SUs return the in-use channels immediately when the PUs' resume the use of the licensed band $=-090-$ Y5E4.

After sending a fragment, the sender waits for a short listen period. During the short listen period, the sender senses the channel to ensure that any PU does not use the channel. After the sender sends three consecutive fragments, it waits for a long listen period. This long listen period is not only for sensing the channel but also for receiving ACK from the receiver. When any PU uses the channel, both the sender and the receiver detect it and stop the current transmission. Further, the receiver broadcasts a specific message of "PU resumes and transmission is not completed" to its neighbors via a control channel. Once the ongoing transmission is aborted owing to PU arrival, the sender negotiates with the receiver to continue the transmission through another channel. 


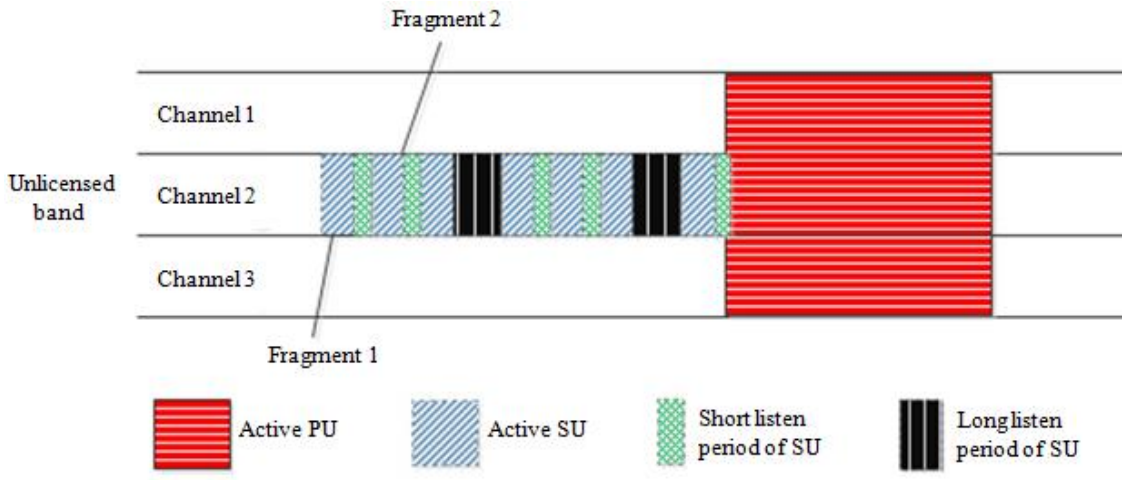

Figure 6. Fragmented Data Transmission

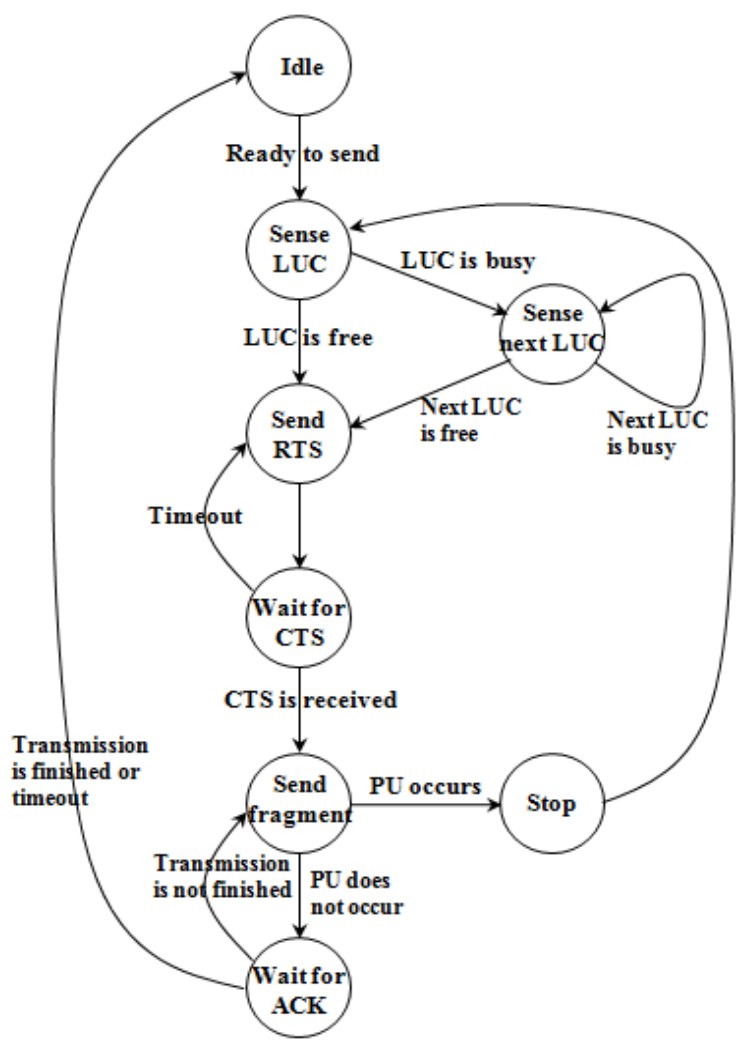

Figure 7. State Transition Diagram at the Sender

The detailed step-by-step operations at the sender and receiver are carried out in accordance with the state transition diagrams shown in Figures 7 and 8, respectively. In Figure 7, LUC stands for the lowest utilized channel. The RTS, fragmented DATA, and ACK frames are directionally transmitted, whereas the CTS frame is transmitted omnidirectionally. When both the sender and the receiver detect the presence of a PU, they stop the ongoing transmission immediately, and the receiver broadcasts the specific message to its neighbors via a control channel as discussed earlier. 


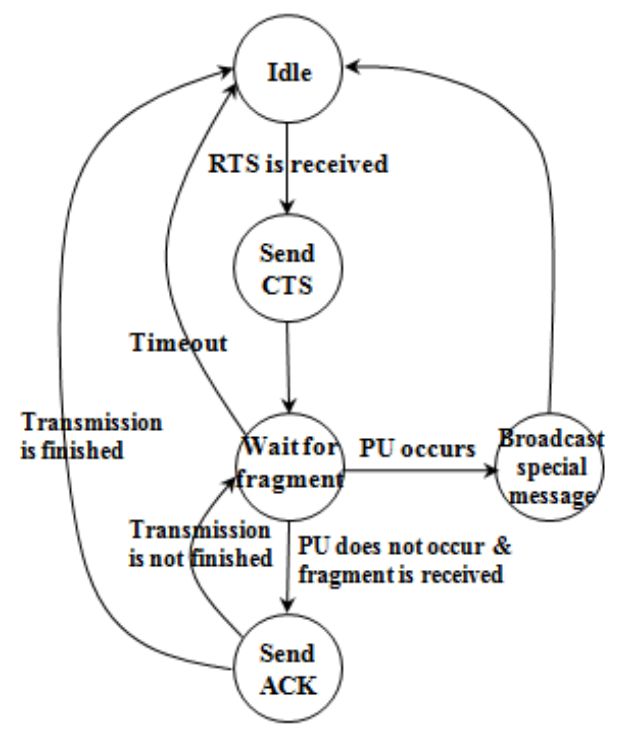

Figure 8. State Transition Diagram at the Receiver

\section{Energy Efficiency Evaluation}

In this section, the energy efficiency of the proposed DC-MAC protocol is evaluated and compared with the conventional protocol in terms of energy consumption per bit. Here, the conventional protocol is CR-aware MAC associated with omnidirectional antennas for CRSNs. As mentioned earlier, to the best of the authors' knowledge, no study on MAC for CRSNs with directional antennas has been proposed in the literature. As a result, the proposed DC-MAC is compared to the conventional MAC protocol for CRSNs with omnidirectional antennas. With the same transmission range, therefore, the energy consumption of DC-MAC is compared with that of the conventional CR MAC.

\subsection{Energy Consumption}

The energy consumption model is based on [19]. When the transceiver starts up, the energy consumption $E_{\text {start }}$ can be calculated by

$$
E_{\text {start }}=P_{L O} \times T_{\text {start }},
$$

where $P_{L O}$ is the total power consumption of the voltage controller oscillator and frequency synthesizer, and $T_{\text {start }}$ is the required setting time.

When the transceiver is in the receiving mode, the energy consumption $E_{r x}$ can be evaluated by

$$
E_{r x}=\left(P_{L O}+P_{R X}\right) \times T_{r x},
$$

where $P_{r x}$ is the power consumption of the low-noise amplifier, mixer, amplifier, and demodulator and $T_{r x}$ is the receiving time. Given the transmission time $T_{t x}$, the transmission energy $E_{t x}$, the power of power amplifier $P_{P A}$, and the power required for transmission $P_{\text {OUT }}$ are expressed as

$$
\begin{aligned}
& E_{t x}=\left(P_{L o}+P_{P A}\right) \times T_{t x}, \\
& P_{P A}=\frac{1}{\eta} \times P_{O U T},
\end{aligned}
$$


and

$$
P_{\text {OUT }}=\mathrm{r}_{\mathrm{PA}} \times r \times d^{n},
$$

respectively, where $\eta$ is the power amplifier efficiency, which varies from $10 \%$ to $70 \%$ depending on the linearity, circuit topology, and technology, $r$ is the data rate, $r_{P A}$ is a constant, $d$ is the transmission distance, and $n$ is the pass loss exponent. In DC-MAC, the sensor nodes are equipped with directional antennas and, thus, $P_{\text {OUT }}$ can be simplified as

$$
P_{\text {DovT }}=\frac{1}{\alpha} \times P_{o U T},
$$

where $\alpha$ is the number of directional antenna beams. When a node finishes transmitting data, it changes into a receive mode.

The energy consumed in changing the mode $E_{\text {switch }}$ can be calculated by

$$
E_{\text {switch }}=P_{L O} \times T_{\text {switch }},
$$

where $T_{\text {switch }}$ is the switching time.

In summary, the total energy consumption in a sensor node $E_{O P}$ can be expressed by

$$
E_{O P}=E_{\text {start }}+E_{t x}+E_{\text {switch }}+E_{r x} \text {. }
$$

\subsection{Simulation Setting}

The performance of DC-MAC is evaluated via MATLAB simulation. Two transceivers-RFM TR 1001 [20], which is operated at $868.35 \mathrm{MHz}$, and the Bluetooth transceiver, which is operated at $2.4 \mathrm{GHz}$ - are used in our simulation. The simulation parameters are listed in Table 1 . For simplicity, the duty cycle is set as 50\%. Note that the duty cycle is the percentage of time that a device spends in the active state over the total time under consideration. For evaluating energy efficiency, data rate is varied from $2 \mathrm{kbps}$ up to $100 \mathrm{kbps}$ in our simulation. Each run of simulation is repeated 100 times and then averaged.

Table 1. Simulation Parameters

\begin{tabular}{|c|c|}
\hline Parameter & Value \\
\hline Number of nodes & 30 \\
\hline Network area & $100 \mathrm{~m} \times 100 \mathrm{~m}$ \\
\hline Transmission range & $20 \mathrm{~m}$ \\
\hline Data rate & $\begin{array}{c}2-100 \mathrm{kbps} \text { for RFM TR 1001 transceiver } \\
2-100 \mathrm{kbps} \text { for Bluetooth transceiver }\end{array}$ \\
\hline Packet length $(L)$ & $100 \mathrm{bits}$ \\
\hline Power amplifier efficiency $(\eta)$ & $100 \mu \mathrm{s}$ \\
\hline Switching time $\left(T_{\text {switch }}\right)$ & 4 \\
\hline $\begin{array}{c}\text { Number of directional antenna } \\
\text { beams }(\alpha)\end{array}$ & $(\alpha 0 \%$ \\
\hline
\end{tabular}




\subsection{Simulation Results}

Figure 9 shows the energy consumption per bit in Joule for DC-MAC and the conventional CR MAC when the RMF TR 1001 transceiver operates at 868.35 MHz. The energy consumption per bit is decreased with the increased data rate. This is mainly because, as the data rate is increased, more data is transmitted for a given period of time and thus the energy per bit is decreased. DC-MAC achieves approximately 50\%-60\% more energy efficiency in comparison to the conventional CR MAC.

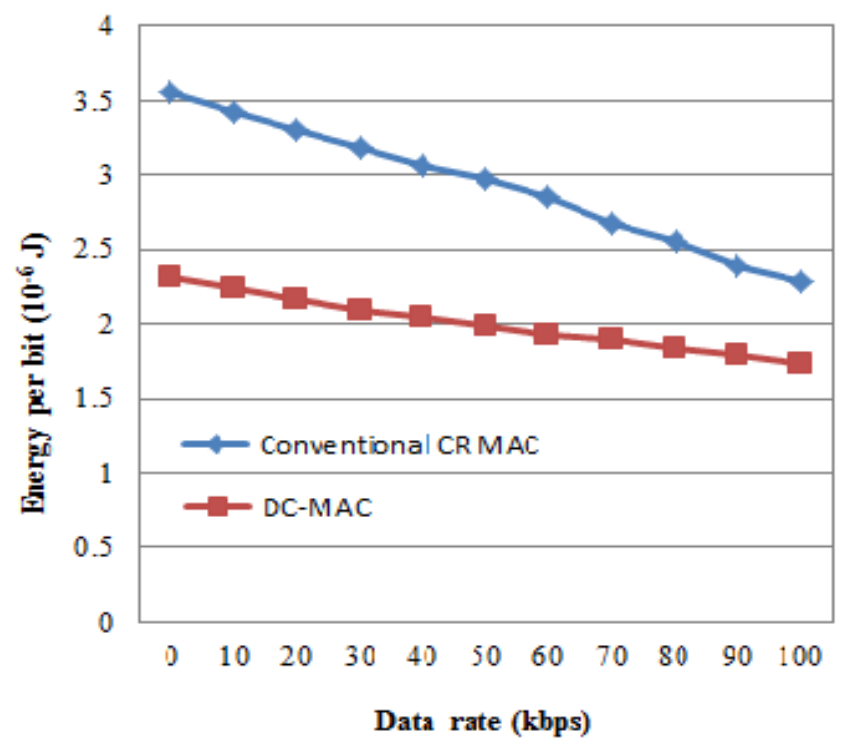

Figure 9. Energy Consumption When Using RMF TR 1001 Transceiver

Figure 10 shows the energy consumption per bit in Joules for DC-MAC and the conventional CR MAC when the Bluetooth transceiver operates at $2.4 \mathrm{GHz}$. The energy consumption per bit drops considerably as the data rate increases. At the low data rate, DC-MAC consumes approximately $60 \%$ less energy in comparison to the conventional CR MAC. Even at the high data rate (e.g., $10 \mathrm{Mbps}$ ), DC-MAC consumes approximately $20 \%$ less energy.

From the simulation results, therefore, it can be easily inferred that the proposed DCMAC is more energy efficient than the conventional approach. This is quite meaningful because the energy efficiency is the most important factor in CRSNs as well as in general WSNs. 


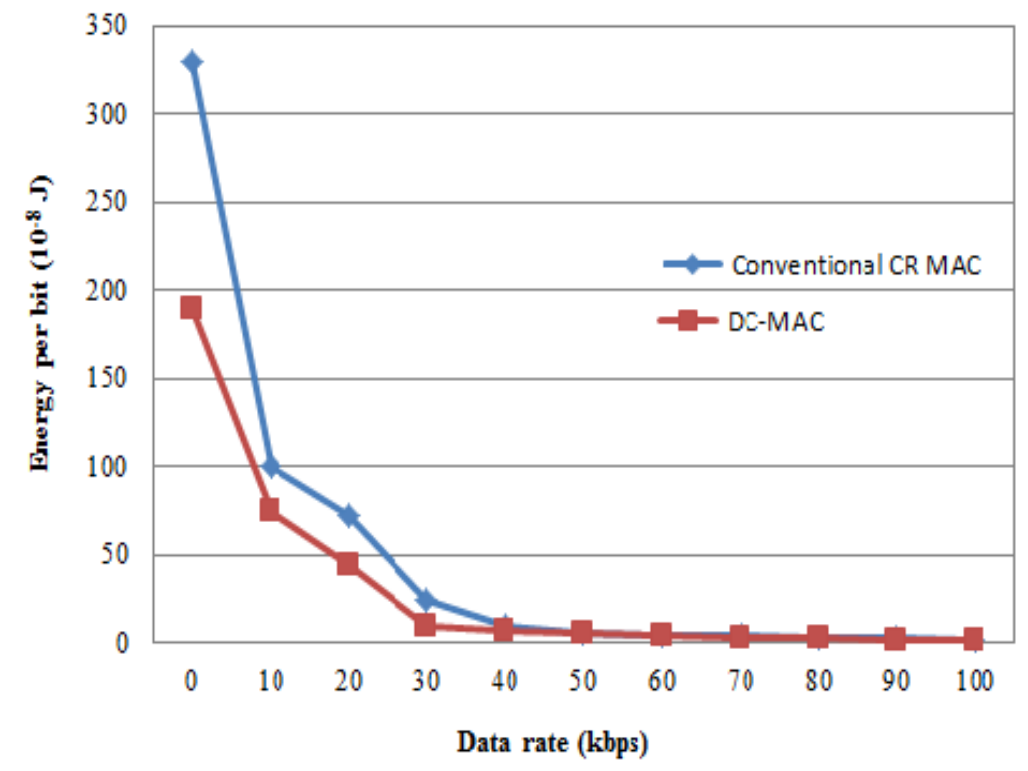

Figure 10. Energy Consumption When Using the Bluetooth Transceiver

\section{Conclusions}

In this paper, an energy-efficient MAC protocol has been proposed for CRSNs using directional antennas. To the best of our knowledge, this is the first work to propose a MAC protocol for CRSNs with directional antennas. According to the simulation study, the proposed DC-MAC is more energy efficient than the conventional protocol, resulting in prolonged network lifetime. In our study, energy efficiency is focused in both design and evaluation because it is the most important design issue in wireless sensor networks. As a future work, however, we will exploit the advantage of directional antennas more aggressively for improving the network throughput.

\section{Acknowledgements}

A preliminary version of this work was presented at the 3rd International Conference on Smart Media and Applications, Dec. 2014 [22]. This work was supported in part by Basic Science Research Program through the National Research Foundation of Korea (NRF) funded by the Ministry of Education (NRF-2013R1A1A2011744). Correspondence should be addressed to Dr. Sangman Moh (smmoh@chosun.ac.kr).

\section{References}

[1] Federal Communications Commission(FCC), Spectrum Policy Task Force Report, http://www.fcc.gov/ spft/files/SEWGFinal_Report_1.pdf, (2002) Nov.

[2] M. H. Islam, C. L. Koh, S. W. Oh, X. Qing, Y .Y .Lai, C. Wang, Y.-C. Liang, B. E. Toh, F. Chin, G. L. Tan, and W. Toh, Spectrum Survey in Singapore: Occupancy Measurements and Analysis, Proceedings of 3rd CROWNCOM, (2008) pp. 1-7.

[3] D. Datla, A. M. Wyglinski, and G. J. Minden, A Spectrum Surveying Framework for Dynamic Spectrum Access Networks, IEEE Transactions on Vehicular Technology, (2009) Vol. 58, no. 8, pp. $4158-4168$.

[4] Y. C. Liang, K. C. Chen, G. Y. Li, and P. Mahonen, Cognitive Radio Networking and Communications: An Overview, IEEE Transactions on Vehicular Technology, (2011) Vol. 60, pp. 3386-3407.

[5] B. Wang and K. J. R. Liu, Advances in Cognitive Radio Networks: A Survey, IEEE Journal of Selected Topics in Signal Processing, (2011) Vol. 5, No. 1, pp. 5-23.

[6] W. Ye, J. Heidemann and D. Estrin, An Energy-Efficient MAC Protocol for Wireless Sensor Networks, Proceedings of IEEE INFOCOM, (2002) Vol. 3, pp. 1567-1576.

[7] Y. Ko, V. Shankarkumar and N. H. Vaudya, Medium Access Control Protocols Using Directional Antennas in Ad Hoc Networks, Proceedings of IEEE INFOCOM, (2000) Vol. 1, pp. 13-21. 
[8] T. V. Dam and K. Langendoen, An Adaptive Energy-Efficient MAC Protocol for Wireless Sensor Networks, Proceedings of SenSys, (2003) pp171-180.

[9] T. Zheng, S. Radhakrishnan, and V. Sarangan, PMAC: An Adaptive Energy-Efficient MAC Protocol for Wireless Sensor Networks, Proceedings of 19th International Parallel and Distributed Processing Symposium (IPDPS), (2005) pp. 237a-237b.

[10] W .R Heinzelman, A. P. Chandrakasan, and H. Balakrishnan, An Application-Specific Protocol Architecture for Wireless Microsensor Networks, IEEE Transactions on Wireless Communications, (2002) pp. 660-670.

[11] T. Shu, S. Cui, and M. Krunz, Medium Access Control for Multi-Channel Parallel Transmission in Cognitive Radio Networks, Proceedings of IEEE GLOBECOM, (2006) pp. 1-5.

[12] H. A. B. Salameh, M. M. Krunz, and O. Younis, MAC Protocol for Opportunistic Cognitive Raidon Networks with Soft Guarantees, IEEE Transactions on Mobile Computing, (2009) Vol. 8, No. 10, pp.1339-1352.

[13] H. Bany Salameh, M. Krunz, and O. Younis, Distance- and Traffic-Aware Channel Assignment in Cognitive Radio Networks, Proceedings of IEEE SECON, (2008) pp. 10-18.

[14] S. Sankaranarayanan, P. Papadimitratos, A. Mishra, and S. Hershey, A Bandwidth Sharing Approach to Improve Licensed Spectrum Utilization, Proceedings of 1st IEEE International Symposium on New Frontiers in Dynamic Spectrum Access Networks, (2005) pp. 279-288.

[15] H. Su and X. Zhang, Cross-Layer Based Opportunistic MAC Protocols for QoS Provisionings over Cognitive Radio Wireless Networks, IEEE Journal on Selected Areas in Communications, (2008) Vol. 26, No. 1, pp.118-129.

[16] Y. Ki and S. J. Yoo, MCR-MAC: Multi-channel Cognitive Radio MAC Protocol for Cooperative Incumbent System Protection in Wireless Ad-hoc Network, Proceedings of Int. Conf. on Ubiquitous and Future Networks, (2009) pp. 6-11

[17] M. A. Shah, G. A. Safdar, and C. Maple, DDH-MAC: A Novel Dynamic De-Centralized Hybrid MAC Protocol for Cognitive Radio Networks, Proceedings of RoEduNet International Conference, (2011) pp.1-6

[18] R. R. Choudhury and N. H. Vaidya, Deafness: A MAC Problem in Ad Hoc Networks When Using Directional Antennas, Proceedings of 12th IEEE Int. Conf. on Network Protocols, (2004) pp.283-292.

[19] A. Wang and C. Sodini, A simple energy model for wireless microsensor transceivers, Proceedings of IEEE Global Telecommunications Conference, (2005) Nov., pp. 3205-3209.

[20] RMF TR 1001 data sheet, Available at http://www.rfm.com/ products/data/tr1001.pdf, (2014).

[21] B. Khatiwada and S. Moh, A Novel Multi-Channel MAC Protocol for Directional Antennas in Ad Hoc Networks, Wireless Personal Communications, (2008) Vol. 80, Issue 3, pp. 1095-1112.

[22] H. Su, C. Baek, and S. Moh, An Energy-Efficient MAC Protocol for Cognitive Radio Sensor Networks Using Directional Antennas, Proceedings of 3rd Int. Conf. on Smart Media and Applications, (2014) Dec., pp. E1.1-E1.4.

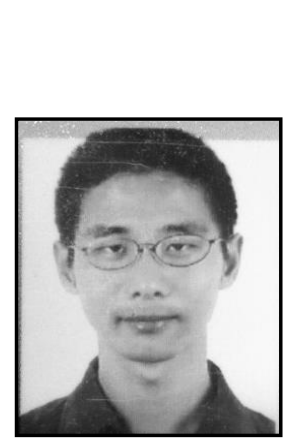

\section{Authors}

Han Su received the B.S. degree in network engineering from Nanjing University of Information Science and Technology, China in 2011 and the M.S. degree in computer engineering from Chosun University, Korea in 2014. Currently, he is an engineer at China Post, Suzhou, China. His current research interests include ad hoc networks and cognitive radio networks with a focus on network architectures and protocols.

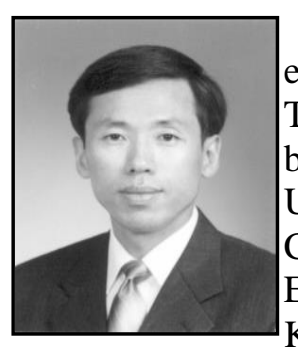

Sangman Moh received the Ph.D. degree in computer engineering from Korea Advanced Institute of Science and Technology (KAIST), Korea in 2002. Since late 2002, he has been a professor in the Dept. of Computer Engineering at Chosun University, Korea. From 2006 to 2007, he was on leave at Cleveland State University, USA. Until 2002, he had been with Electronics and Telecommunications Research Institute (ETRI), Korea, where he served as a project leader, since he received the M.S. degree in computer science from Yonsei University, Korea in 1991. His research interests include mobile computing and 
networking, ad hoc and sensor networks, cognitive radio networks, and parallel and distributed computing systems. He has published more than 200 papers in international and domestic journals and conference proceedings, and has held more than 40 overseas and domestic patents. He serves on the program committees of international conferences and workshops in his areas of interest. Dr. Moh is a member of the IEEE, the ACM, the IEICE, the KIISE, the IEIE, the KIPS, the KICS, the KMMS, the IEMEK, the KISM, and the KPEA. 